\title{
Infrared Holography for Wavefront Reconstruction and Interferometric Metrology
}

\author{
Sergio De Nicola, Andrea Geltrude, \\ Massimiliano Locatelli, Kais Al-Naimee, \\ Riccardo Meucci and F.Tito Arecchi \\ Istituto Nazionale di Ottica - CNR Firenze
}

Italy

\section{Introduction}

Long wavelength interferometry has been widely applied in different fields, such as infrared optics, infrared transmitting materials, high-reflective multilayer dielectric coatings for highpower laser systems. In optical metrology, long-wave interferometers are also employed for shape measurement of reflective rough surfaces and for testing optical systems that requires deep aspherics. An advantage of using longer wavelength is that the aspheric departure from the best fit reference-sphere, in unit of the probing wavelength, is reduced at longer wavelength, thus allowing one to obtain an interferogram of the deep aspheric under test. This leads to extension of the unambiguous distance measurement range, a well-known problem in interferometric metrology, where the essential difficulty relates to the interferometric fringe order, which cannot be determined unambiguously from a single measurement of phase interference. This problem is also of particular significance in digital holographic and interferomety based applications where digital processing of the recorded interferogram makes it possible to extract quantitatively amplitude and phase of the numerically reconstructed wavefront (Cuche el al., 1999a, 1999b; Vest, 1979; Yaroslavsky \& Eden, 1996). Digital holography based imaging techniques provide real time capabilities to record also three-dimensional objects using interference between an object wave and a reference wave captured by an image sensor such a CCD sensor. Three-dimensional 3D information of the object can be obtained from the numerical reconstruction of a single digitally recorded hologram, since the information about the optically interfering waves is stored in the form of matrices. The numerical reconstruction process offers many more possibilities than conventional optical processing (Goodman \& Lawrence, 1967; Stetson \& Powell, 1966; Stetson \& Brohinsky, 1985). For example, it is possible to numerically focus on any section of the three dimensional volume object without mechanical focusing adjustment (Grilli et al., 2001; Lai et al., 2000), correct optical components defects such as lens aberrations or compensate the limited depth of field of an high magnification microscope objective. Full digital processing of holograms requires high spatial resolution sensor arrays with demanding capabilities for imaging applications and non destructive testing. In this regard, several new recording materials and optoelectronic sensors have been devised for 
recording holograms beyond the visible wavelengths, in the infrared. Coherent imaging based on digital processing of infrared holograms presents the great advantage of providing quantitative data from the recorded interference pattern (Lei et al., 2001a, 2001b; Leith \& Upatnieks, 1965; Nilsson \& Carlsson, 2000; Seebacker et al., 2001) but, the lower spatial resolution of the infrared sensor arrays compared to those working in the visible region, appears to be a major limiting factor to accurate numerical wavefront reconstruction for the digitized interferogram.

In this chapter we will describe potential applications of digital processing methods for whole optical wavefront reconstruction of infrared recorded holograms. The basic principle of numerical reconstruction of digitized holograms will be reviewed and we will discuss numerical methods to compensate the loss of spatial resolution at longer wavelength and the limited spatial resolution of the recording array We will present several examples to show that infrared digital holography can be exploited as a high accuracy technique for testing both reflective and transmissive objects. In order to demonstrate the feasibility of digital holography in the infrared region we will describe a two beam interferometric technique for recording holograms, both in reflection and transmission type geometry which employs a pyroelectric sensor array. The availability of pyroelectric sensor arrays for recording digital infrared holograms offers the advantage of a compact and efficient interferometric set-up for recording infrared hologram. We will also present application of digital holographic processing methods for numerical reconstruction of wavefronts of optical beams, such as Laguerre-Gauss beams possessing wavefront singularities in the infrared region. In fact, this technique can be usefully employed for characterizing the vorticity of infrared beams of potential use in optical telecommunication applications, where the preservation of the purity of the mode along the propagation direction is a problem of crucial importance. We will present examples of characterization of the vortex signature of infrared Laguerre-Gauss beam through the numerical reconstruction of the singular phase of the beam from the digitized hologram.

\section{Theory and principle of operation of digital holography}

The principle of the optical recording and reconstruction in classical holography is illustrated in Fig.1. The reference beam $R$ interferes at the plane of the holographic plate at off-axis angle $\theta$ with respect to the object beam $O$. In this set-up the reconstructed image is spatially separated from the zero-order diffraction and the second image, the so-called 'twin image'. These three diffraction orders propagate along different directions and can be observed separately, leading to a significant improvement compared to the original in-line configuration originally developed by Gabor (Gabor, 1948; Goodman, 1996) where the zeroorder and the two conjugate images overlap.

The intensity distribution $I(x, y)$ across the $x-y$ holographic recording plane can be written as the modulus squared of the complex superposition $O(x, y)+R(x, y)$, namely

$$
I(x, y)=|O(x, y)+R(x, y)|^{2}=|R(x, y)|^{2}+|O(x, y)|^{2}+R^{*}(x, y) O(x, y)+R(x, y) O^{*}(x, y)
$$

where the symbol * denotes complex conjugation, $O(x, y)=|O(x, y)| \exp \left[i \varphi_{0}(x, y)\right]$ is the complex amplitude of the object wave with real amplitude $|O(x, y)|$ and phase $\varphi_{0}(x, y)$ and 
$R(x, y)=|R(x, y)| \exp \left[i \varphi_{R}(x, y)\right]$ is the complex amplitude of the reference wave with real amplitude $|R(x, y)|$ and phase $\varphi_{R}(x, y)$. Eq. (1) can be modified to the form

$$
I(x, y)=\left(|R(x, y)|^{2}+|O(x, y)|^{2}\right)+2|R(x, y)||O(x, y)| \cos \left(\varphi_{o}(x, y)-\varphi_{R}(x, y)\right)
$$

which shows explicitly that the recorded hologram contains a term with an amplitude and phase modulated spatial carrier. For the numerical reconstruction of the recorded hologram, the interference pattern $I(x, y)$ is illuminated by the reference wave $R(x, y)$, i.e, we have

$$
R(x, y) I(x, y)=R(x, y)|R(x, y)|^{2}+R(x, y)|O(x, y)|^{2}+|R(x, y)|^{2} O(x, y)+R^{2}(x, y) O^{*}(x, y)
$$

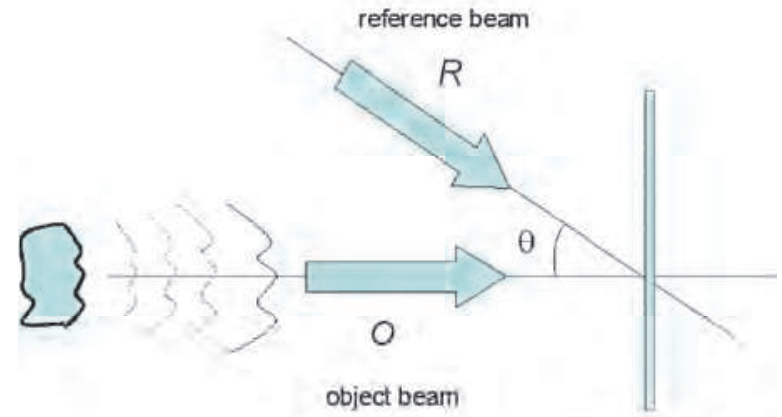

hologram plate

a)

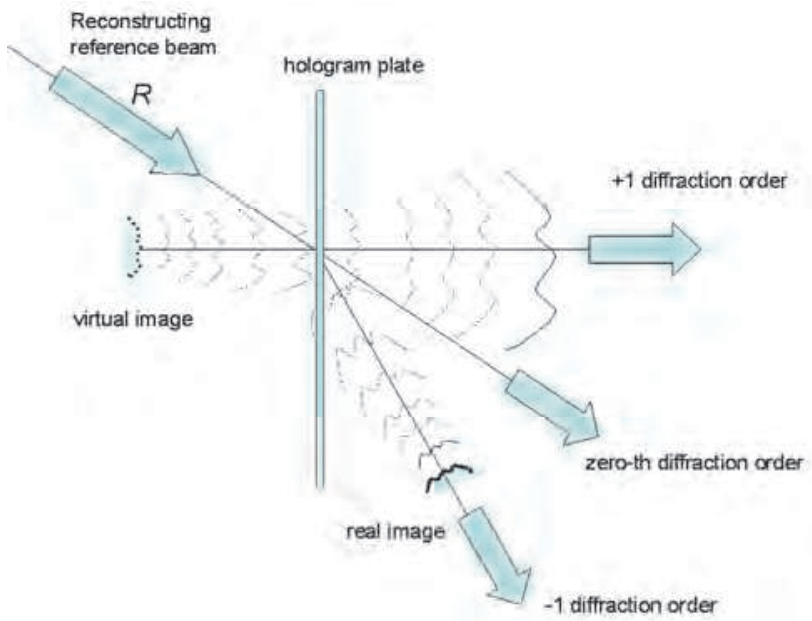

b)

Fig. 1. Optical configuration for recording (a) and for reconstruction (b) of off-axis holograms. 
The first term on the right side of this equation is proportional to the reference wave field, the second one is a spatially varying "cloud" surrounding the first term. These two terms constitute the zero-order of diffraction or DC term. The third term represents, apart for a constant factor, an exact replica of the original wavefront $O(x, y)=|O(x, y)| \exp \left(i \varphi_{o}(x, y)\right)$ and for this reason it is called virtual image, or simply image of the object. The last term is another copy, the so called twin image of the original object wave or real image.

In holography the hologram can be regarded as an amplitude transmittance that diffracts the reference wave. In DH the object wave field is determined through the numerical calculation of the optical field propagation of $R(x, y) h(x, y)$ from the holographic plane back to the object plane $\xi, \eta$. The numerical reconstruction of a digitally recorded hologram follows the scalar diffraction theory in the Fresnel approximation of the RayleighSommerfield diffraction integral. The reconstructed diffracted field $Q(\xi, \eta)$ in the reconstruction plane $\xi, \eta$ at distance $d$ from the hologram plane can be written in the paraxial approximation in the following form

$$
Q(\xi, \eta)=\frac{1}{i \lambda d} \exp \left(i \frac{2 \pi}{\lambda} d\right) \int_{-\infty}^{\infty} \int_{-\infty}^{\infty} R(x, y) I(x, y) \exp \left[i \frac{\pi}{\lambda d}\left((x-\xi)^{2}+(y-\xi)^{2}\right)\right] d x d y
$$

Eq.(3) is the starting point for reconstructing numerically the digitized hologram in the paraxial approximation, where the $x$ and $y$ values and the corresponding $\xi$ and $\eta$ values in the reconstruction plane are small compared to the distance $d$ (see Fig.2).

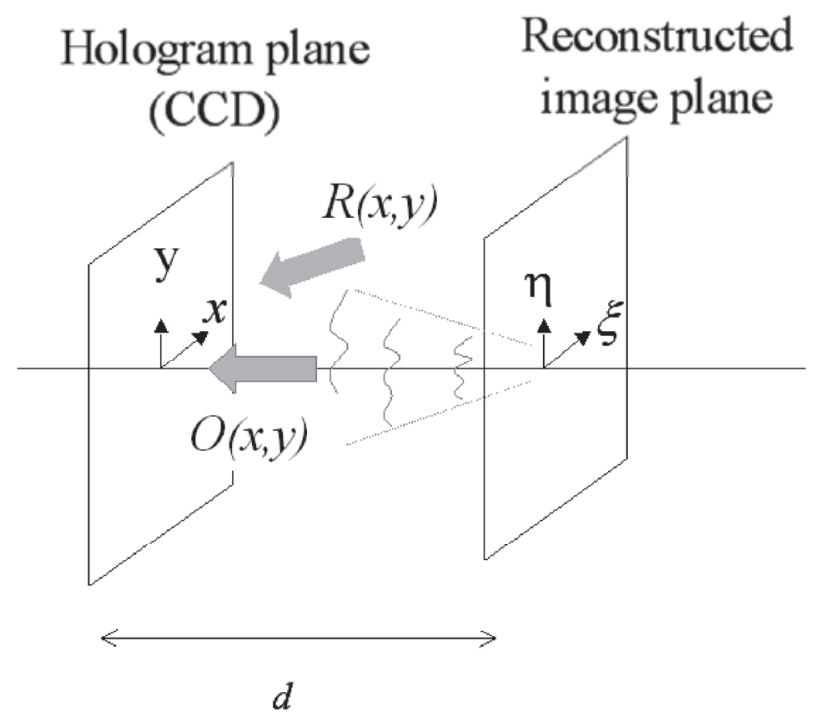

Fig. 2. Optical set-up in off-axis digital holography

Once the complex field $Q(\xi, \eta)$ has been calculated at distance $d$, the intensity $I(x, y ; d)$ and phase distribution $\varphi(x, y ; d)$ of the reconstructed image can be determined by the following relations 


$$
\begin{gathered}
I(x, y ; d)=|Q(x, y)|^{2} \\
\varphi(x, y ; d)=\arctan \frac{\operatorname{Im}[Q(x, y)]}{\operatorname{Re}[Q(x, y)]}
\end{gathered}
$$

Eq. (4b) provides phase values wrapped in the interval $[\pi, \pi]$. A well-known unwrapping procedure can be employed to convert the phase modulo- $2 \pi$ into a continuous phase distribution in order to obtain a smooth phase image.

\subsection{Reconstruction methods}

In the following we will describe the main methods for numerical reconstruction of holograms (Kreis \& Jueptner, 1997; Kronrod et al., 1972; Schnars \& Jueptner, 1994, 2002).

\subsubsection{Fresnel transformation method (FTM)}

The convolution integral given by Eq.(3) can be manipulated to obtain the reconstructed diffracted field $Q(\xi, \eta)$ in terms of the so-called Fresnel transformation of the hologram function. Indeed it results that

$$
\begin{aligned}
& Q(\xi, \eta)=\frac{1}{i \lambda d} \exp \left(i \frac{2 \pi}{\lambda} d\right) \exp \left[i \frac{\pi}{\lambda d}\left(\xi^{2}+\eta^{2}\right)\right] \\
& \int_{-\infty}^{\infty} \int_{-\infty}^{\infty} R(x, y) I(x, y) \exp \left[i \frac{\pi}{\lambda d}\left(x^{2}+y^{2}\right)\right] \exp \left[-i \frac{2 \pi}{\lambda d}(\xi x+y \eta)\right] d x d y
\end{aligned}
$$

Eq.(5) shows that the reconstruction field is determined essentially by the two-dimensional Fourier transformation of the multiplication of the hologram $I(x, y)$ by the reference wave $R(x, y)$ and the chirp phase function

$$
w(x, y)=\exp \left[i \frac{\pi}{\lambda d}\left(x^{2}+y^{2}\right)\right]
$$

Eq. (5) can be written in terms of the Fourier integral

$$
Q(\xi, \eta)=\frac{1}{i \lambda d} \exp \left(i \frac{2 \pi}{\lambda} d\right) \exp \left[i \frac{\pi}{\lambda d}\left(v_{\xi}^{2}+v_{\eta}^{2}\right)\right]\left(F^{+1}[R(x, y) I(x, y) w(x, y)]\left(v_{\xi}, v_{\eta}\right)\right)
$$

where the direct $(+1)$ or inverse $(-1)$ continuous two dimensional Fourier transformations of the function $f(x, y)$ are defined, respectively, by

$$
F^{ \pm 1}[f(x, y)]\left(v_{\xi}, v_{\eta}\right)=\int_{-\infty}^{\infty} \int_{-\infty}^{\infty} f(x, y) \exp \left[\mp i 2 \pi\left(v_{\xi} x+v_{\eta} y\right)\right] d x d y
$$

In Eq. (7) $v_{\xi}$ and $v_{\eta}$ are the spatial frequencies (Kreis, 2002a, 2002b) corresponding to the spatial variables $\xi$ and $\eta$ in the reconstruction plane and they are related to the reconstruction wavelength $\lambda$ and to the reconstruction distance $d$ by the following relations

$$
v_{\xi}=\frac{\xi}{\lambda d} \quad v_{\eta}=\frac{\eta}{\lambda d}
$$


With the off-axis geometry the object wave and the reference wave arrive in the hologram plane with separate directions and, according to the above equations, the different terms of the numerically reconstructed wavefront propagate along different directions, owing to their different spatial frequencies. In fact, if Eq.(2) is substituted into Eq. (5), it is clear that the reconstruction of the DC term, the virtual and the real image are essentially governed by the frequency content of the respective spectra at the reconstruction distance $d$, which ultimately impose restrictions on the spatial bandwidth of the object and reference beam. If the reference field is given by $R(x, y)=\sqrt{I_{R}} \exp \left[i\left(k_{x} x+k_{y} y\right)\right]$ where $I_{R}=|R(x, y)|^{2}$ is the intensity of the reference field and $\mathbf{k}=\left(k_{x}, k_{y}, k_{z}\right)$ is the corresponding wave vector, the three terms are separated in the Fourier domain corresponding to the reconstruction plane $\xi, \eta$ at distance $d$. The zero-order is located around the origin while the image and the twin image are symmetrically centred on $\left(k_{x} / 2 \pi, k_{y} / 2 \pi\right)$ and $\left(-k_{x} / 2 \pi,-k_{y} / 2 \pi\right)$, respectively. To achieve good quality reconstruction in DH, the sampling theorem (Nyquist criterion) has to be fulfilled across the whole CCD array area. The criterion requires at least two pixels per fringe period and this implies that the maximum interference angle $\alpha_{\max }$ between the spherical wavelet from each point of the object and the reference wave field is determined by the pixel size $\Delta x$ according to the relation

$$
a_{\max }=\frac{\lambda}{2 \Delta x}
$$

Relation (9) expresses the fact that for recording an hologram by a CCD array with pixel spacing $\Delta x$ at least two pixels per fringe are needed. For example in case of a camera with pixel size $\Delta x=9 \mu \mathrm{m}$, the maximum interference angle is $\alpha_{\max } \approx 1.7^{\circ}$ for $\lambda=532 \mathrm{~nm}$. Mathematically, the two-dimensional spatial sampling $I(n \Delta x, m \Delta y)$ of the hologram $I(x, y)$ on a rectangular raster of $N \times M$ points can be described by the following relation

$$
I(n \Delta x, m \Delta y)=I(x, y) \operatorname{rect}\left(\frac{x}{N \Delta x}, \frac{y}{M \Delta y}\right) \sum_{n=1}^{N} \sum_{m=1}^{M} \delta(x-n \Delta x, y-m \Delta y)
$$

where $\delta(x, y)$ is the two-dimensional Dirac-delta function, $n$ and $m$ are integer numbers, $N \Delta x \times M \Delta y$ is the area of the digitized hologram and rect $(x, y)$ is equal to one if the point of coordinated $(x, y)$ is inside the area of the digitized hologram, and is zero elsewhere. $\Delta x$ and $\Delta y$ in Eq.(10) are the distances between the neighboring pixels on the CCD array in the horizontal and vertical directions, respectively. If the whole CCD array has a finite width given by $N \Delta x \times M \Delta y$, where $N$ and $M$ are the pixel numbers in each directions, the discrete representation of the Fresnel reconstruction integral given by Eq. (5) can be written as

$$
\begin{aligned}
Q\left(r \Delta v_{\xi}, s \Delta v_{\eta}\right)= & \frac{1}{i \lambda d} \exp \left(i \frac{2 \pi}{\lambda} d\right) \exp \left[i \pi \lambda d\left(r^{2} \Delta v_{\xi}^{2}+s^{2} \Delta v_{\eta}^{2}\right)\right] \\
& \times \Delta x \Delta y \sum_{n=-N / 2}^{N / 2-1} \sum_{m=-M / 2}^{M / 2-1} I(n \Delta x, m \Delta y) R(n \Delta x, m \Delta y) w(n \Delta x, m \Delta y) \exp \left[i 2 \pi\left(\frac{r n}{N}+\frac{s m}{M}\right)\right]
\end{aligned}
$$


Eq. (11) can allows to compute a matrix of $N \times M$ complex numbers of the reconstructed field via the discrete two-dimensional fast Fourier transform algorithm. According to the theory of discrete Fourier transform, the sampling frequency intervals are given by $\Delta v_{\xi}=1 / N \Delta x$ and $\Delta v_{\eta}=1 / M \Delta y$ which, together with relations (8) allow us to determine the dimensions $\Delta \xi \times \Delta \eta$ of the reconstruction pixel, namely

$$
\Delta \xi=\frac{\lambda d}{N \Delta x} \quad \Delta \eta=\frac{\lambda d}{M \Delta y}
$$

According to Eq. (12) the pixel width in the reconstructed plane is different from those of the digitized hologram and it scales inversely to the aperture of the optical system, i.e., to the side length $S=N \Delta x$ of the hologram (limiting the analysis to the $x$-direction for the sake of simplicity). This result is in agreement with the theory of diffraction which predicts that a distance $d$ from the hologram plane the developed diffraction pattern is characterized by the diameter $\lambda d / S$ of its Airy disk (or speckle diameter). Therefore the resolution of the reconstructed image (amplitude or phase image) is limited by the diffraction limit of the imaging system through the automatic scaling imposed by the Fresnel transform. Assuming that the hologram $I(x, y)$ has spatial frequencies smaller than those in the quadratic phase factor $w(x, y)$, the main problem when calculating Eq. (11) comes from an adequate sampling of the exponential function $w(x, y)$ inside the integral and of the global phase factor $\exp \left[i \pi \lambda d\left(r^{2} \Delta v_{\xi}^{2}+s^{2} \Delta v_{\eta}^{2}\right)\right]$ multiplying the expression in Eq.(11). Assuming the sampling of $w(x, y)$ in the Nyquist limit, it is easy to obtain the approximate condition (limiting our analysis to one dimension only) that determines the range of distances $d$ where the discrete Fresnel reconstruction algorithm (cfr. Eq (11)) gives good results, namely

$$
d \geq d_{c}=\frac{N \Delta x^{2}}{\lambda}
$$

The same argument can be applied to the global phase factor $\exp \left(i \pi \lambda d r^{2} \Delta v_{\xi}^{2}+s^{2} \Delta v_{\eta}^{2}\right)$, which has too rapid variation with increasing spatial distance $d$, giving, this time, the condition $d \leq d_{c}$. A good reconstruction of both the amplitude and phase-contrast image is accomplished only if the equality $d=d_{c}$ is assumed, while, if only the amplitude-contrast reconstruction is considered, the less restrictive condition $d \geq d_{c}$ must hold. In fact, in this case the global phase factor is unessential for evaluating intensity distribution and the intensity profiles are of lower spatial frequency variation than the corresponding phase. Note that the size $\Delta \xi_{c}$ of the reconstruction pixel at distance $d=d_{c}$ in the Nyquist limit coincides with pixel size of the sampled hologram, i.e., $\Delta \xi_{c}=\lambda d_{c} / N \Delta x=\Delta x$. As an example, for $N=512$ pixel, $\lambda=632 \mathrm{~nm}$ and pixel size $\Delta x=\Delta y=11 \mu \mathrm{m}$, the Fresnel method is valid for distance greater than $98 \mathrm{~mm}$; for $N=1024$ pixel, $\lambda=532 \mathrm{~nm}$ and $\Delta x=\Delta y=6.7 \mu \mathrm{m}$, the distance has to be greater than $24.5 \mathrm{~mm}$. From Eq. (12) it can be easily deduced that the width $S_{I}=N \Delta \xi$ of the numerical reconstruction increases linearly with reconstruction distance $d$ according to the scaling law

$$
S_{I}=\frac{\lambda d N}{S}
$$


where $N$ and the width $S=N \Delta x$ of the hologram are input parameters in the reconstruction process. Nevertheless this result is only compatible with condition given by Eq.(13), derived from the appropriate sampling of reconstructed amplitude image. This means that maintaining the number $N$ as a constant may lead to a badly sampled reconstructed image if the reconstruction distance does not satisfy the above requirements. By padding the recorded hologram with zeros in the border, after Fresnel diffraction the external part of the reconstructed hologram can be prevented from leaving the support matrix and entering the opposite side of the matrix because of aliasing. This is of particular importance in the numerical reconstruction of off-axis recorded holograms where the use of the pixel area of the recording array is less efficient.

\subsubsection{Convolution transformation method}

An alternative way of numerical reconstruction of holograms is through the calculation of the propagated angular spectrum, the so called "convolution approach" to digital holography. The reconstructed field, in the paraxial approximation, can be written in this case in the following form

$$
Q\left(\left(v_{\xi}, v_{\eta}\right)\right)=\exp \left(i \frac{2 \pi}{\lambda} d\right) \mathrm{F}^{-1}\left\{\left(\exp \left[-i \pi \lambda d\left(v_{\xi}^{2}+v_{\eta}^{2}\right)\right] F^{+1}[R(x, y) h(x, y)]\left(v_{\xi}, v_{\eta}\right)\right\}\right.
$$

where the Fourier transform of the chirp function $w(x, y)$ given by Eq. (4) has been used, namely

$$
F^{+1}[w(x, y)]\left(v_{\xi}, v_{\eta}\right)=i d \lambda \exp \left[\left[-i \pi \lambda d\left(\left(v_{\xi}^{2}+v_{\eta}^{2}\right)\right]\right)\right]
$$

It can be shown that when the angular spectrum is used, the use of two Fourier transforms for computing Eq.(15), once for taking the Fourier transform of the hologram (multiplied by the reference wave) and another time for taking the inverse Fourier transform, leads to a cancellation of the scale factor between the input and output field to obtain that the pixel size of the reconstructed images equal to that of the sampled hologram, i.e., $\Delta \xi=\Delta x$ and $\Delta \eta=\Delta y$ and the actual sizes of $\mathrm{h}$ the input hologram and reconstructed image are identical $\left(S_{I}=S\right)$. We point out that, although Eq.(6) and Eq.(15) are formally equivalent, the different use of the DFT algorithm to perform the calculation of the same diffraction integral, makes the convolution-based algorithm valid for near distances $d \leq d_{c}$. Both methods overlap at distance $d=d_{c}$. Clearly this method is computationally more expensive than the direct evaluation of the Fresnel integral, since it requires two Fourier transforms (one direct and one inverse) but it is advantageous for keeping constant the length scales of the reconstructed images for all distances satisfying the near-field approximation (Zhang et al., 2004). From the discrete complex values of the reconstructed field, the intensity $I(r, s ; d)$ and phase distribution $\varphi(r, s ; d)$ of the reconstructed image can be determined according to the following two equations

$$
\begin{gathered}
I(r, s ; d)=|Q(r \Delta \xi, s \Delta \eta)|^{2} \\
\varphi(r, s ; d)=\arctan \frac{\operatorname{Im}[Q(r \Delta \xi, s \Delta \eta)]}{\operatorname{Re}[Q(r \Delta \xi, s \Delta \eta)]}
\end{gathered}
$$




\subsection{Digital holographic interferometry}

The fringe pattern that results from the interference of the reference beam and an object beam carries phase information on the object under test and any change in its state gives rise to a corresponding modification in the phase information.

If the complex fields $Q\left(\xi, \eta ; s_{1}\right)$ and $Q\left(\xi, \eta ; s_{2}\right)$ are the numerical reconstructions of two holograms recorded at different states $s_{1}$ and $s_{2}$ of the object, the corresponding phase change $\Delta \phi\left(\xi, \eta ; s_{2}-s_{1}\right)$ is given by

$$
\Delta \phi\left(\xi, \eta ; s_{2}-s_{1}\right)=\operatorname{Arg}\left[Q\left(\xi, \eta ; s_{2}\right)\right]-\operatorname{Arg}\left[Q\left(\xi, \eta ; s_{1}\right)\right]
$$

by taking into account that

$$
\operatorname{Arg}[Q(\xi, \eta, s)]=\arctan \left[\frac{\operatorname{Im}(Q(\xi, \eta, s))}{\operatorname{Re}(Q(\xi, \eta, s))}\right]
$$

we can express the phase change in the following form

$$
\Delta \phi\left(\xi, \eta ; s_{2}-s_{1}\right)=\frac{\operatorname{Re}\left[Q\left(\xi, \eta ; s_{2}\right)\right] \operatorname{Im}\left[Q\left(\xi, \eta ; s_{1}\right)\right]-\operatorname{Re}\left[Q\left(\xi, \eta ; s_{2}\right)\right] \operatorname{Im}\left[Q\left(\xi, \eta ; s_{1}\right)\right]}{\operatorname{Re}\left[Q\left(\xi, \eta ; s_{2}\right)\right] \operatorname{Re}\left[Q\left(\xi, \eta ; s_{1}\right)\right]+\operatorname{Im}\left[Q\left(\xi, \eta ; s_{2}\right)\right] \operatorname{Im}\left[Q\left(\xi, \eta ; s_{1}\right)\right]}
$$

For instance, in the case of deformation measurement, $s_{1}$ and $s_{2}$ are states of deformation of the object under investigation and the calculated interference phase provides information about the displacement field onto the surface in case of opaque objects or the full optical path variation occurring in transparent objects (Rastogi, 1994). Digital Holographic interferometry has also been applied to measure deformations of either large and very small objects, to investigate the refractive index changes (Dubois et al., 1999; Zito et al., 2009) and for comparing accurately the shapes of objects and for removing spherical aberrations introduced by high numerical aperture objective lenses employed in digital holographic microscopy applications where the paraxial approximations implicit in the Fresnel treatment often fails (Jueptner et al, 1987; Kato et al., 2003; Kim, 2000; Pedrini et al., 2001).

\section{Infrared digital holography}

In this section we present some applications of digital holography and of the previously described wavefront reconstruction technique in the infrared region as a high accuracy technique for testing reflective objects. The method makes use of a two-beam interferometric set-up (De Nicola et al., 2008) for recording digital off-axis interferograms of reflective objects at wavelength $10.6 \mu \mathrm{m}$. A scheme of the set-up is shown in Fig. 3.

A conventional gas flowing $\mathrm{CO}_{2}$ laser emitting on the $\mathrm{P}(20)$ line at $\lambda=10.6 \mu \mathrm{m}$ is employed as an infrared source. The laser cavity, $82 \mathrm{~cm}$ long, is defined by a partially reflective flat mirror $(\mathrm{R}=95 \%)$ and an out-coupling mirror $(\mathrm{R}=90 \%)$ of $3 \mathrm{~m}$ radius of curvature mounted upon a piezoelectric translator. The output laser beam is horizontally polarized by means of an intracavity ZnSe Brewster window. The system, pumped by an electric discharge of $10 \mathrm{~mA}$, when the laser threshold is approximately $9 \mathrm{~mA}$ provides an output optical power in the range of $500-800 \mathrm{~mW}$. The spatial profile of the laser is set in the fundamental $\mathrm{TEM}_{00}$ Gaussian mode by means of an intra-cavity iris diaphragm. In this configuration, the laser 


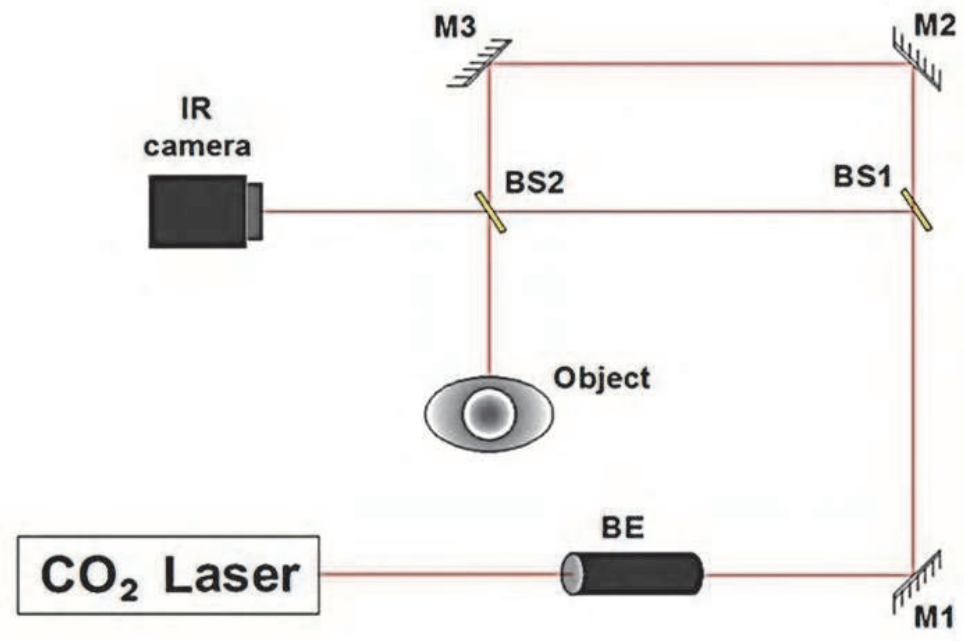

Fig. 3. Mach-Zehnder interferometer employed to record IR digital holograms of reflective samples: M1, M2 and M3, mirrors; BE beam expander; BS1 and BS2, beam splitters.

beam is characterized by a spot size of $6 \mathrm{~mm}$ on the flat mirror and a divergence less than $2 \mathrm{mrad}$. Figure 3 shows the DH optical set-up based on a Mach-Zehnder interferometer. The infrared beam is directed to a beam expander with magnification $2.5 \times$ and $15 \mathrm{~mm}$ diameter of output beam. Two mirrors M1 and M2 and two beam splitters, BS1 (70T/30R at $45^{0}$ angle of incidence ) and BS2 (50T/50R at 450 angle of incidence), are used to form the interferometer. The beam splitters are ZnSe coated windows with a diameter $50 \mathrm{~mm}$ each. The interferometer allows to record the interference patterns between the two beams, the reference beam and that reflected by the test object on the detection plane of a pyroelectric videocamera_Spiricon Pyrocam III, Model PY-III-C-A, which has a matrix of $N \times N=124 \times$ 124 pyroelectric sensor elements of $\mathrm{LiTaO} 3$, with square pixel size $85 \mu \mathrm{m} \times 85 \mu \mathrm{m}$ and centerto-center spacing of the pixels, the pixel pitch, $100 \mu \mathrm{m} \times 100 \mu \mathrm{m}$. The reference beam interferes with the object at a small angle $\alpha \leq \lambda / 2 \Delta \xi=3^{\circ}$, as required by the sampling theorem. The pyroelectric camera allows detection of $\mathrm{CW}$ infrared laser radiation by means of an internal chopper. It is connected to a personal computer to record digitized fringe patterns. The reflective objects used are two opaque aluminium blocks. The first one is a rectangle of size $20 \mathrm{~mm} \times 35 \mathrm{~mm}$ that has letters inscribed. The letters are "UOR" and "XUO" ( about $3 \mathrm{~mm} \times 4 \mathrm{~mm}$ each). The second one is a disc of radius $25.4 \mathrm{~mm}$ which has inscribed a set of concentric circular tracks. The aluminium blocks are located at distance $d=250 \mathrm{~mm}$ from the array. The discrete finite form of Eq. (11) is obtained through the pixel pitch $\Delta \xi \times \Delta \eta=100 \mu m \times 100 \mu m$ of the pyrocam array and the reconstructed object field $\psi(x, y ; d)$ is obtained by applying the 2D fast Fourier transform (2D-FFT) algorithm to the discrete samples of the quantity $I(\xi, \eta) R(\xi, \eta) \exp \left[i \pi\left(\xi^{2}+\eta^{2}\right) / \lambda d\right]$. By the 2D-FFT algorithm, the size of the reconstruction pixel at distance $d$ is $\Delta x \times \Delta y=d \lambda / N \Delta \xi \times d \lambda / N \Delta \eta$. For a reconstruction distance $d=250 \mathrm{~mm}$, the resolution of the reconstructed field distribution is limited by the size $\Delta x \times \Delta y=213 \mu m \times 213 \mu m$ of the reconstruction pixel. To 
compensate for the loss of resolution with increasing reconstruction distance $d$, the digitized hologram was padded with value of zero to achieve a larger array of $N^{*} \times N^{*}=256 \times 256$ points and a reconstruction pixel of size $103 \mu \mathrm{m} \times 103 \mu \mathrm{m}$ at a distance $d=250 \mathrm{~mm}$. This process increases the number of 2D-FFT points, changing artificially the period, while holding the spatial sampling rate of the digitized hologram fixed. In this manner, spatial spectral components of the reconstructed images, originally hidden from view can be shifted to points where they can be observed. However aliasing created by the use of a 2DFFT may result from spreading or leakage of the spectral components away from the correct frequency leading to undesirable modification of the reconstructed image. Aliasing occurs during numerical implementations if the reconstruction distance is less than the minimum object-to-hologram recordable distance $d_{\min }=N^{*} \Delta \xi^{2} / \lambda$. Increasing the size $N^{*}$ of the window function tends to increase $d_{\min }$. The condition $d>d_{\min }$ sets the maximum number of samples with value of zero at the end of the digitized holograms for a given hologram recording distance. In our case, for the infrared wavelength $\lambda=10.6 \mu \mathrm{m}$, the condition is satisfied since we have $d_{\min }=241 \mathrm{~mm}$ for $N^{*}=256$. The reconstruction was obtained with a reference beam of the form $R(\xi, \eta)=\exp \left[i\left(k_{x} \xi+k_{y} \eta\right)\right]$ where the two components $k_{x}$ and $k_{y}$ of the wave-vectors were adjusted to centre the image in the reconstruction plane. The determined phase values are wrapped (Yamaguchi \& Zhang, 1997; Yamaguchi et al., 2002) in the interval $[-\pi, \pi]$. A well-known unwrapping procedure can be employed to convert the phase modulo- $2 \pi$ into a continuous phase distribution in order to obtain a smooth 3-D phase profile (Demoli et al., 2003; Gass et al., 2003; Schnars, 1994). The height distribution $h(x, y)$ of the object is the information to be retrieved. It is related to the reconstructed phase distribution $\varphi(\xi, \eta ; d)$ by the simple relationship $h(x, y)=\lambda \varphi(x, y ; d) / 4 \pi$. Fig.(4) shows the results of the reconstruction of the image of the letters inscribed in the first aluminium block. Amplitude images were reconstructed from the digitized infrared holograms according to method discussed in the previous section. In Fig.4(a), 4(c) the size of the reconstruction pixel is $213 \mu \mathrm{m} \times 213 \mu \mathrm{m}$; Fig. 4(b), 4(d) shows the corresponding reconstructions with padding operations.

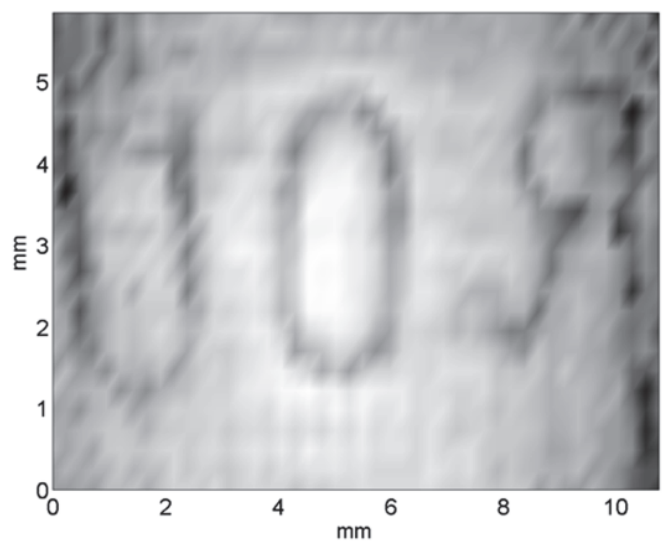

a) 


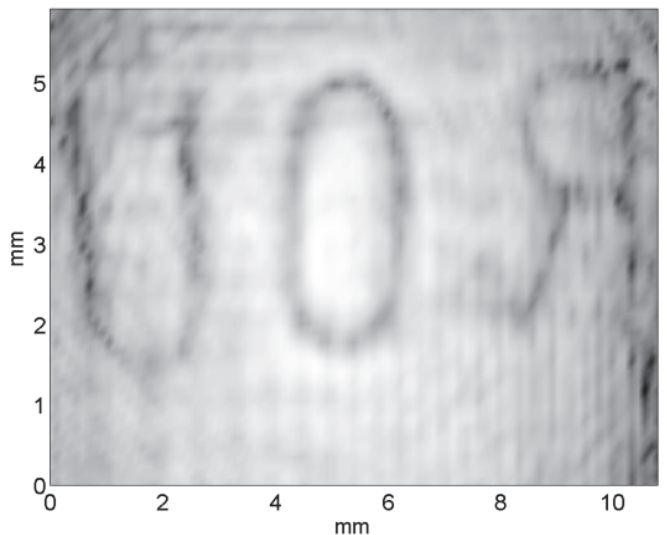

b)

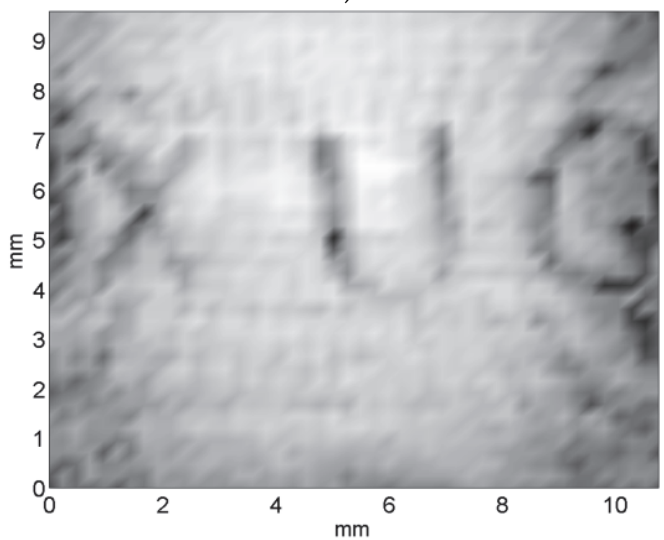

c)

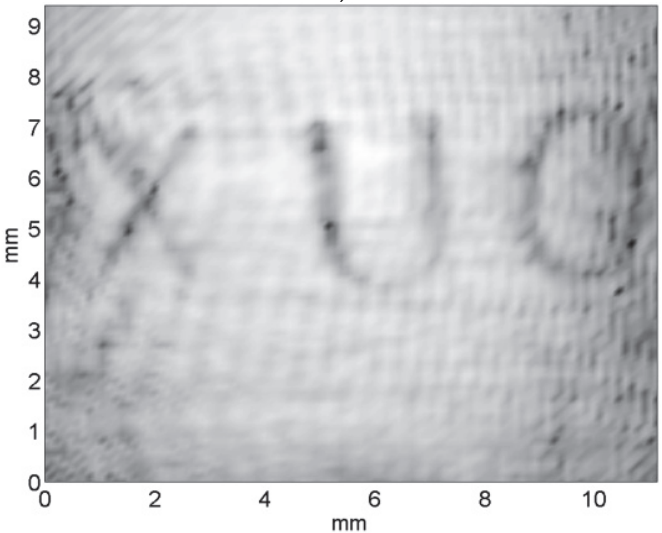

d)

Fig. 4. Amplitude reconstruction of the letters "ROU" before padding operation (a), after zero padding $(\mathrm{b})$; “XUO” before padding operation (c), after padding $(\mathrm{d})$. 
The size is $103 \mu \mathrm{m} \times 103 \mu \mathrm{m}$, and the resolution is clearly improved, as it can be seen by comparing the reconstructed images shown Fig. 4(b), 4(d) with those of Fig. 4(a), 4(c). The hologram of the aluminium disk shaped object is shown in Fig. 5. The concentric circular tracks superimposed on the interference fringes are clearly visible.

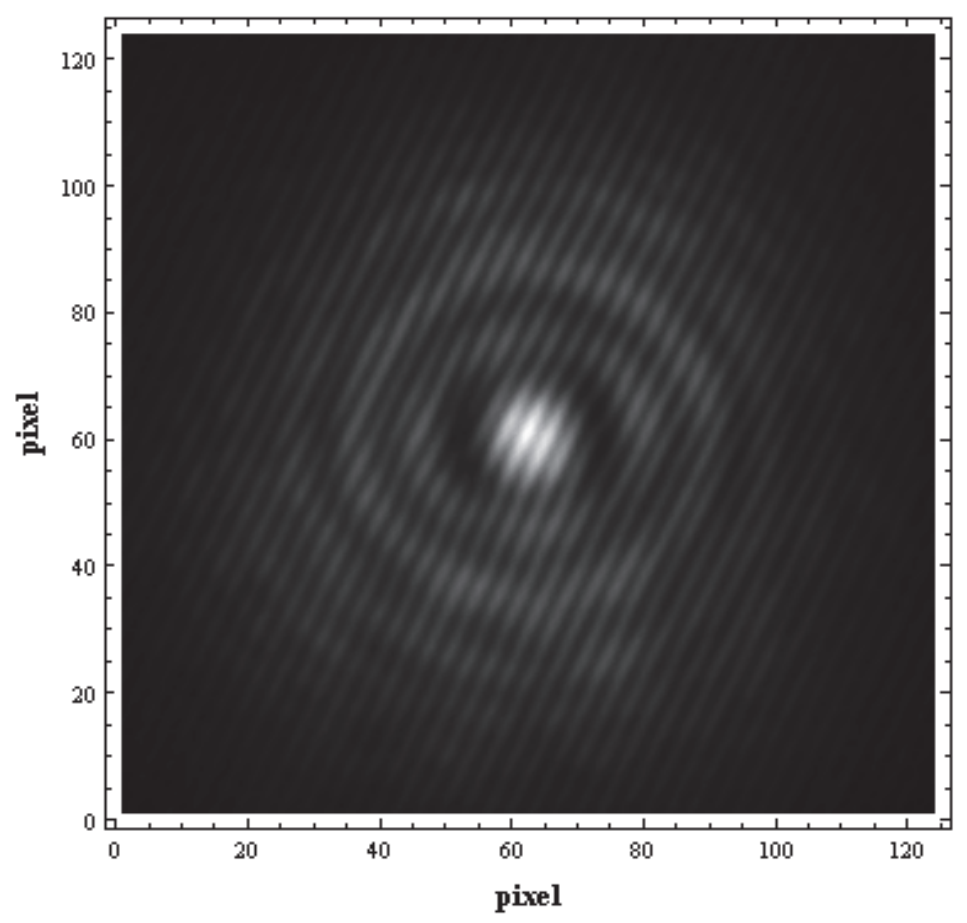

Fig. 5. Infrared hologram of an aluminium disk shaped object which has inscribed a set of concentric circular tracks.

Figure (6) shows the phase images reconstructed form the disk shaped object. Fig. 6(a), 6(c) and $6(\mathrm{e})$ display the phase distribution of the object reconstructed without padding operation and Fig. 6(b), 6(d) and 6(f) show the corresponding reconstructions with zero padding operation. It is noticeable in the $3 \mathrm{D}$ perspectives plots of the reconstructed phase shown in Fig. 6(d) and 6(f) that the circular-shaped tracks inscribed in the steel disk are better resolved with padding operation. 


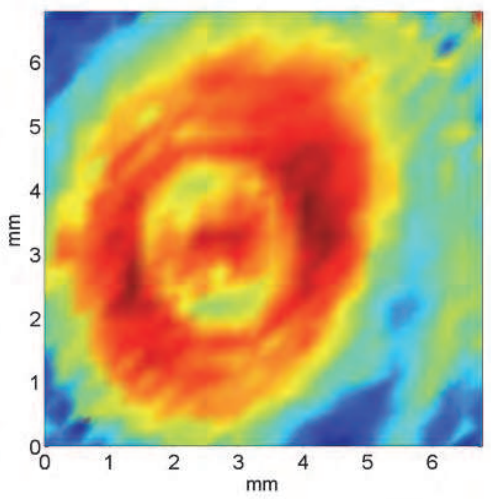

a)

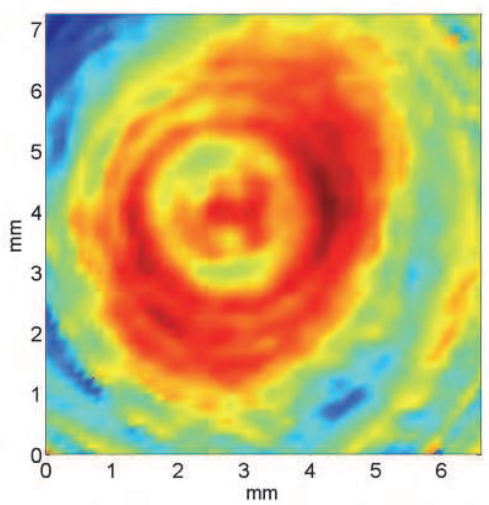

b)

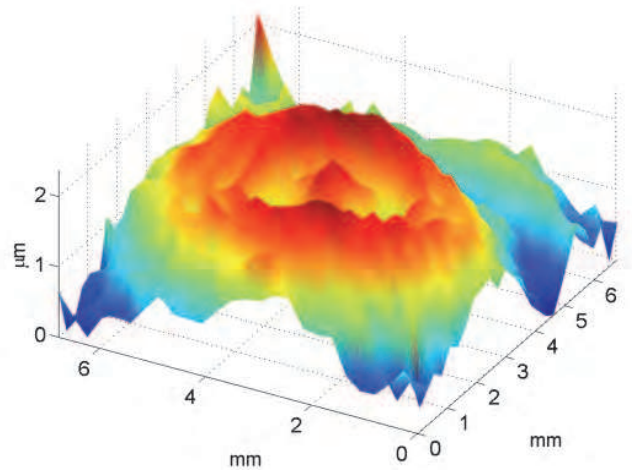

c) 


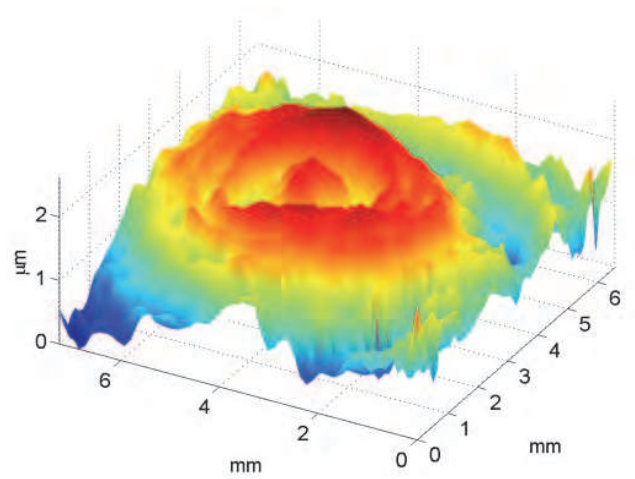

d)

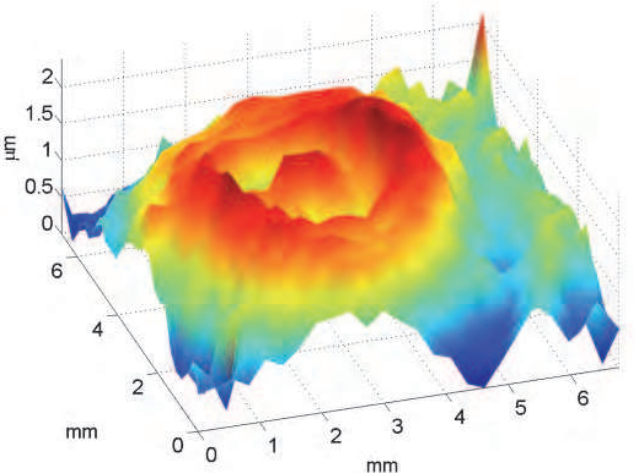

e)

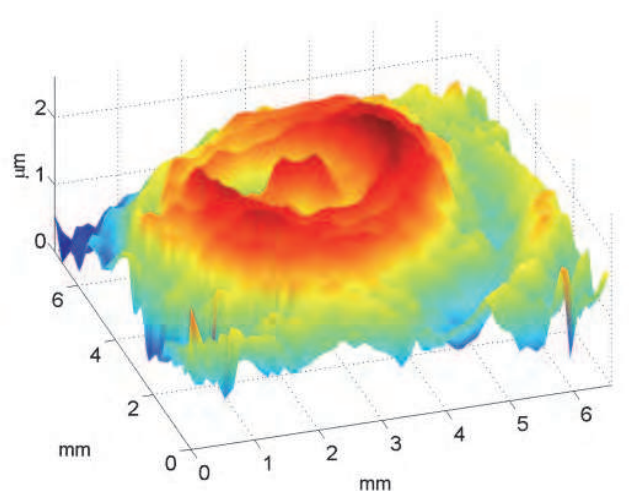

f)

Fig. 6. Phase images of aluminium disc:(a) two-dimensional reconstruction without padding operation; (b) 2D reconstruction with padding; (c), (e) two different 3D maps of the reconstructed surface profile without padding; (d), (f) with zero padding. 


\subsection{Large object investigation}

Whenever large object recording and reconstruction is needed, digital holography with $\mathrm{CO}_{2}$ laser (Allaria et al., 2003) has many advantages, with respect to digital holography in the visible region. First of all, a long wavelength radiation has a lower sensitivity to sub micrometric vibrations and this peculiarity provides a higher fringe visibility when large samples are investigated. A second advantage is related to the high output power of $\mathrm{CO}_{2}$ lasers allowing to irradiate more efficiently and uniformly the surface of large samples (Pedrini et al.,1996). The last advantage derives directly from Eq. 9. Working with a longer wavelength, it is possible to use larger angles and, ultimately, smaller distances between the object and the recording device. In Fig. 7, a configuration to realize Infrared Digital Holography (IDH) of large objects is shown (Pelagotti et al., 2010). In this case we used a $\mathrm{CW} \mathrm{CO}_{2}$ laser emitting in the TEM 00 mode at $10.6 \mu \mathrm{m}$ with $110 \mathrm{~W}$ output power. Interferometric patterns where recorded by means of an ASi microbolometric infrared camera with a resolution of $640 \times 480$ pixels and $25 \mu \mathrm{m}$ pixel pitch. In this configuration the object is placed at the minimum distance allowed by Eq. 9. In this set up the laser beam is first divided by a ZnSe beam splitter $\left(\mathrm{BS}_{1}\right)$ which reflects $90 \%$ of the impinging radiation and transmits the remaining $10 \%$. The transmitted beam, which constitutes the reference beam, after passing through a variable attenuator (VA), is enlarged by a ZnSe spherical lens of 1 inch focal length $\left(\mathrm{L}_{2}\right)$ in order to reach the thermocamera with a suitable low intensity. The object beam is enlarged by means of a spherical ZnSe lens $\left(L_{1}\right)$ of 1 inch focal length and directly sent to the object under investigation.

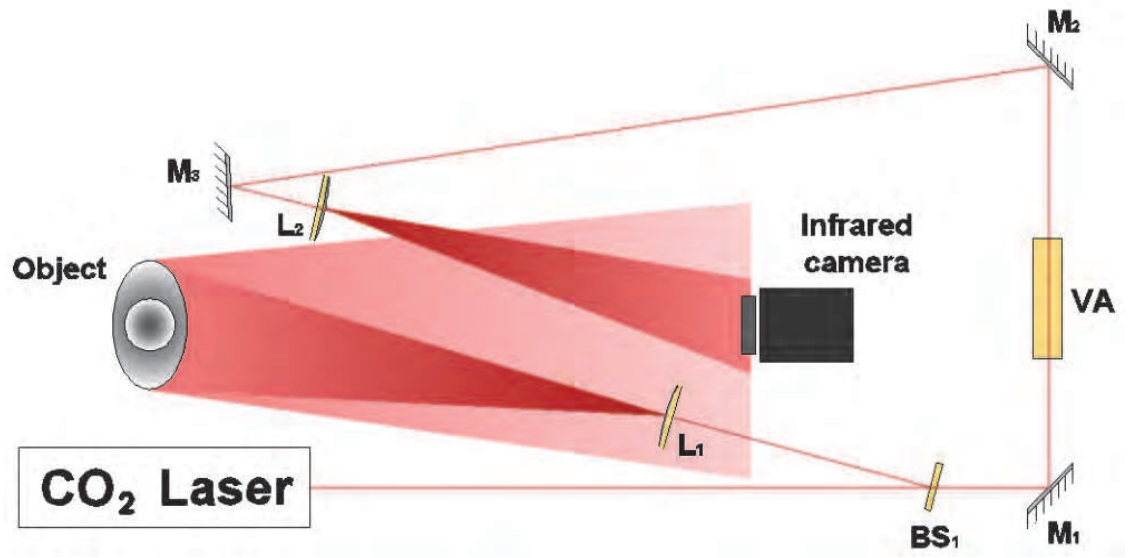

Fig. 7. Single beam setup: $M_{1}, M_{2}$ and $M_{3}$ are plane mirrors; $B_{1}$ is a $Z n S e$ 90/10 beam splitter; $L_{1}$ and $L_{2}$ are $Z n S e$ spherical lenses 1in focal length; the object is a plastic mannequin $1.90 \mathrm{~cm}$ high.

The speckle interferogram, whose visibility is controlled by means of the variable attenuator (VA), is collected by the recording device. With this configuration we recorded holograms of the bronze statue $30 \mathrm{~cm}$ high shown in Fig. 8(a) (Paturzo et al., 2010). The hologram and its numerical amplitude reconstruction are shown in Fig 8(b), Fig 8(c), respectively. 


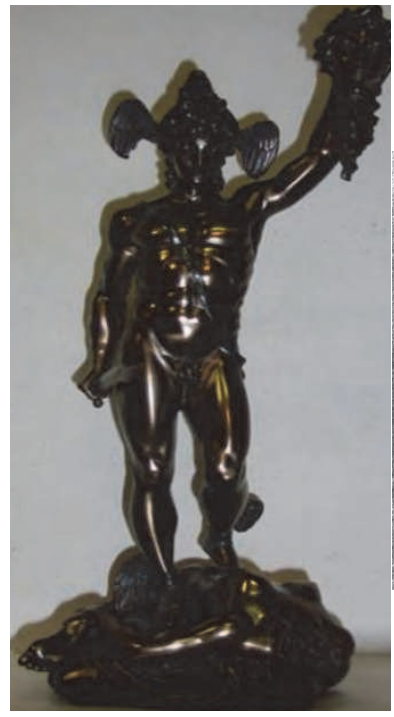

(a)

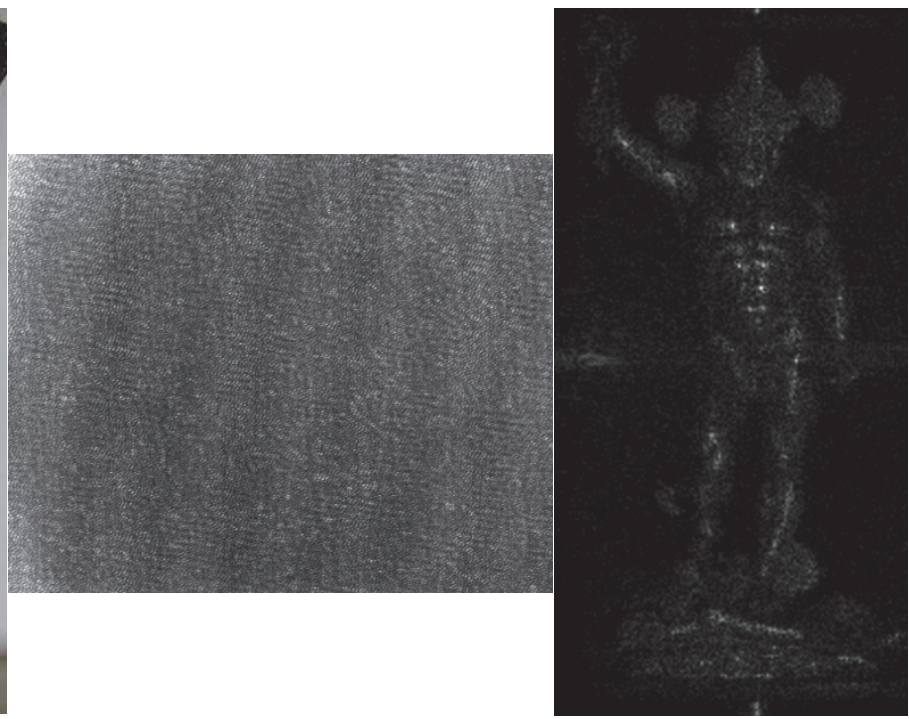

(b)

(c)

Fig. 8. (a) Photo of the Perseus statue; (b) acquired interferogram; (c) numerical reconstruction.

\section{Digital holography for wavefront reconstruction of infrared Laguerre- Gaussian modes}

In this section we present some applications of digital holography for analyzing the spatial distribution and signature vorticity of Laguerre-Gaussian (LG) modes. LG modes have been largely employed for interesting application such as optical trapping, rotational frequency shift and optical manipulation of micrometric systems (Allen et al., 1992, 1999; Arecchi et al., 1991). The concept of singularities in electromagnetic fields possessing an orbital angular momentum (Soskin \& Vasnetov, 2001; Leach et al., 2002) has an extensive literature because of their interesting properties and potential applications. Laguerre-Gaussian beams are examples of optical fields, that possess wave-front singularities (optical vortices) of topological charge $\ell$, where $\ell$ can take any integer value, that are related to the azimuthal angular dependence $\exp (i \ell \phi)$ in the transverse distribution of the optical field. The azimuthal mode index $\ell$ (orbital helicity) is physically related to OAM, $\ell \hbar$ per photon, of the LG modes. The LG modes of azimuthal index $\ell$ and radial index $p$ are

$$
\begin{aligned}
\psi_{\ell p}(x, y, z) & =\sqrt{\frac{2 p !}{\Pi(\ell+p) !}} \frac{1}{w(z)}\left(\frac{r \sqrt{2}}{w(z)}\right)^{\ell} \exp \left(-\frac{r^{2}}{w(z)^{2}}\right) \times \\
& L_{p}^{\ell}\left(\frac{2 r^{2}}{w(z)^{2}}\right) \exp \left(-\frac{i k r^{2}}{2 R(z)}\right) \exp (-i \ell \phi) \times \\
& \exp \left[-i(2 p+\ell+1) \arctan \left(\frac{z}{z_{r}}\right)\right],
\end{aligned}
$$


where $r$ is the radial coordinate, $\phi=\arctan (y / x)$ the azimuthal coordinate and $L_{p}^{\ell}(x)$ are the generalized Laguerre polynomials with azimuthal and radial mode numbers $\ell$ and $p$, respectively. The production and characterization of free space Laguerre-Gaussian beams at comparatively longer wavelengths gives the possibility of using optical vortices for observing the transfer of angular momentum to relatively larger objects, since, for a fixed power, the angular momentum in the beam is proportional to the wavelength. Quantitative characterizations of the structure and signature of the vorticity of infrared LG beams can be obtained by using an interferometer for recording the interference pattern of the LG mode (the object beam) and an external plane-wave reference beam (De Nicola et al., 2010). The experimental set-up is shown in Fig. 9.

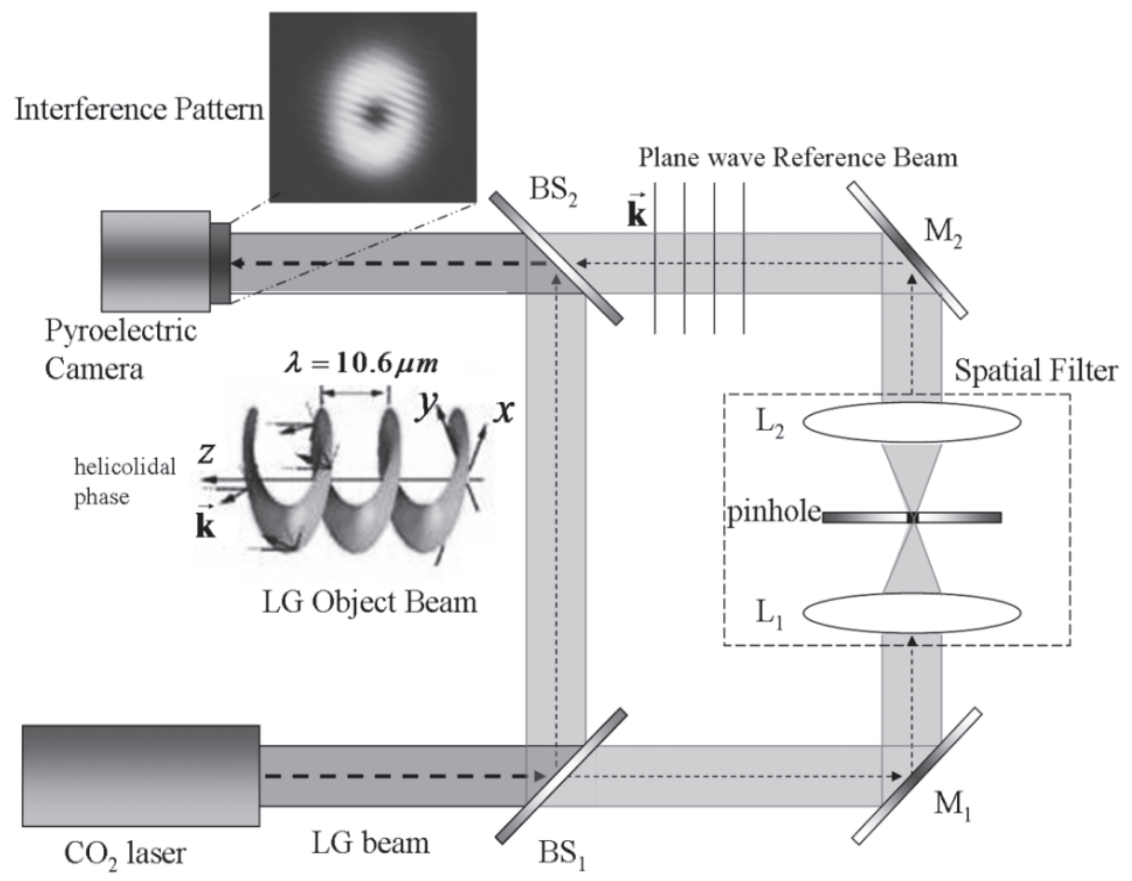

Fig. 9. Outline of the Mach-Zehnder interferometric setup. $\mathrm{A} \mathrm{CO}_{2}$ laser source operating at the wavelength $\lambda=10.6 \mu \mathrm{m}$ produces an LG mode $\psi_{10}$, divided at the $\mathrm{BS}_{1}$ (beam splitter) position into an object beam (LG mode) and a reference beam. The latter is a plane wave beam obtained after having spatially filtered and expanded the vortex field with the collimating lenses and pinhole system L1-P- $\mathrm{L}_{2} . \mathrm{M}_{1}$ and $\mathrm{M}_{2}$ are mirrors. The object beam interferes with the reference beam at the beam splitter $\mathrm{BS}_{2}$ position. The interference pattern is recorded with an IR pyroelectric camera.

A vortex optical field in the mid infrared range is produced by inserting a circular diaphragm of wide aperture (Fresnel number larger than one) inside the optical cavity of a $\mathrm{CO}_{2}$ laser. This operation easily leads to laser emission in the doughnut mode TEM ${ }_{01}^{*}$,but the optical field sometimes presents impurities originating from the residual contributions 
of the fundamental mode or higher order cavity modes. The $\mathrm{CO}_{2}$ laser source operates at the wavelength $\lambda=10.6 \mu \mathrm{m}$. The laser cavity is defined by a partially reflective flat mirror $(\mathrm{R}=95 \%)$ and an out-coupling mirror $(\mathrm{R}=90 \%)$ mounted upon a piezoelectric translator. As aforementioned, the LG mode is selected with a diaphragm and is horizontally polarized by means of an intracavity ZnSe Brewster window.

The interferometric configuration comprises two mirrors, indicated as $\mathrm{M}_{1}$ and $\mathrm{M}_{2}$, and two beam splitters, $\mathrm{BS}_{1}\left(70 \mathrm{~T} / 30 \mathrm{R}\right.$ at $45^{\circ}$ angle of incidence) and $\mathrm{BS}_{2}\left(50 \mathrm{~T} / 50 \mathrm{R}\right.$ at $45^{\circ}$ angle of incidence) as depicted in figure 9. The output laser beam is a linear superposition of $\mathrm{TEM}_{10}$ and TEM ${ }_{01}$ Hermite-Gaussian modes (the doughnut mode $\mathrm{TEM}^{*}{ }_{01}$ ) corresponding to $\ell=1$

(a)

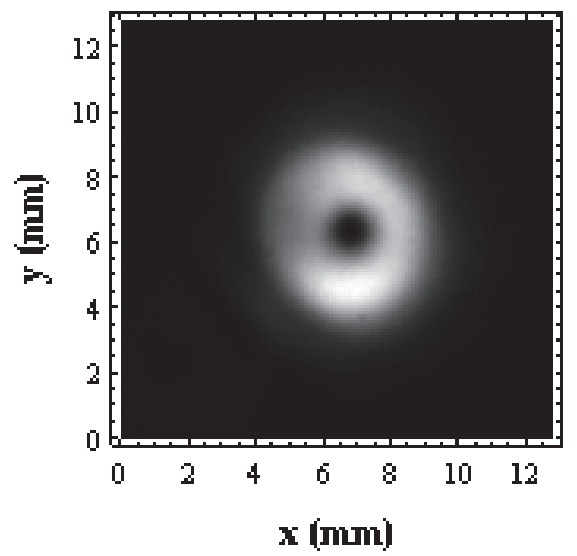

(c)

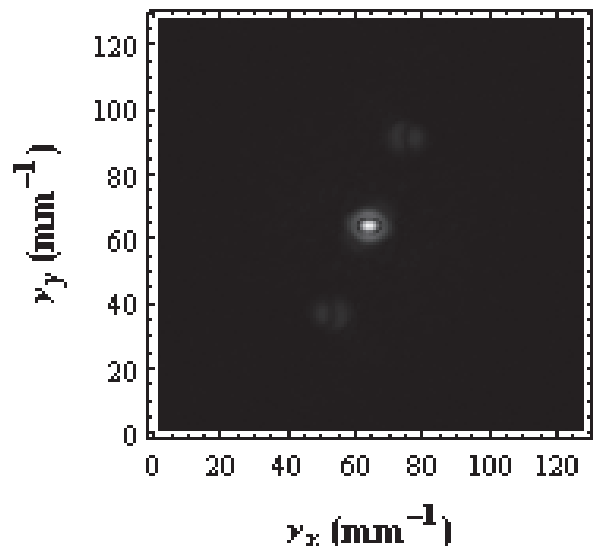

(b)

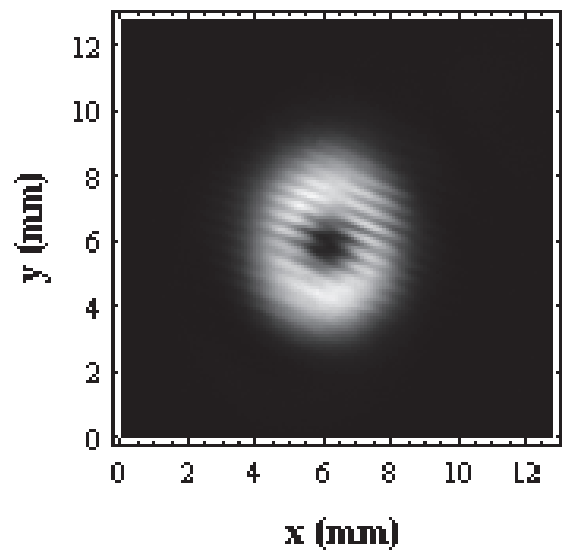

(d)

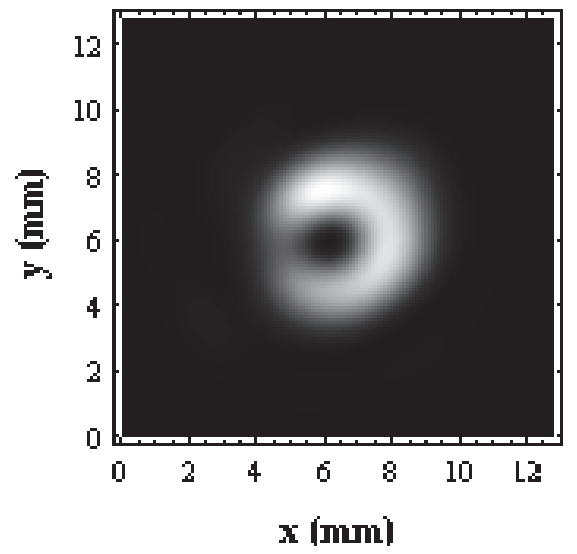

Fig. 10. Infrared Laguerre-Gaussian beam analysis based on Fresnel reconstruction of offaxis digital hologram: (a) intensity distribution of the LG mode $\psi_{10}$ recorded at the IR camera plane; (b) digitized interference intensity profile between LG object beam and plane wave reference; (c) Fourier transform of the carrier modulated interference pattern; (d) reconstructed vortex beam amplitude 
lenses $\left(\mathrm{L}_{1}\right.$ and $\left.\mathrm{L}_{2}\right)$ of focal length $f=3 \mathrm{~cm}$ and a pinhole $(\mathrm{P})$ of $250 \mu \mathrm{m}$ placed in between the two mirrors $\mathrm{M}_{1}$ and $\mathrm{M}_{2}$, as shown in Fig. 9. The two beam splitters $\left(\mathrm{BS}_{1}\right.$ and $\mathrm{BS}_{2}$ ) are $\mathrm{ZnSe}$ and $p=0$, the lowest order vortex, with a beam waist $\mathrm{w}_{o}=2.4 \mathrm{~mm}$ at the out-coupling mirror position. The LG mode $\psi_{10}$ represents the object beam. The reference beam consists of a plane wave obtained with the spatial filter/expansion system $\mathrm{L}_{1} \mathrm{PL}_{2}$ that comprises two coated windows with a diameter of $50 \mathrm{~mm}$. The vortex output beam is split into reference and object beams at the $\mathrm{BS}_{1}$ position. The interference pattern between the plane wave reference and the object beam is recorded on the detection plane of an internally chopped pyroelectric video camera (Spiricon Pyrocam III Model PY-III-C-A), having an infrared sensor of $124 \times 124$ pixel elements of $\mathrm{LiTaO}_{3}$ with square size of $85 \mu \mathrm{m}$ and a pixel pitch $\Delta x=100 \mu \mathrm{m}$. The reference beam interferes with the object beam at a small angle $\left(a \leq \lambda /(2 \Delta x) \sim 3^{\circ}\right)$ as required by the sampling theorem. In Fig. 10(a) we can see the intensity distribution of the LG vortex beam $\psi_{10}(\ell=1$ and $p=0)$ with a dark central core, recorded by the pyroelectric array at distance $d=52 \mathrm{~cm}$ from the out-coupling mirror of the cavity. The digitized carrier modulated infrared interference pattern $I_{10}(x, y)$ in the $(x, y)$-plane of the camera is presented in Fig. 10(b).

Because of the off-axis recording geometry, the two-dimensional Fourier transform of the fringe pattern is characterized by three dominant diffraction orders, as shown in Fig. 10(c) where the amplitude of the Fourier transform has been represented. The full complex two-dimensional Fourier transform of the fringe pattern can be written in the form

$$
\begin{aligned}
\tilde{I}_{10}\left(v_{x}, v_{y}\right)=\tilde{I}_{d c}\left(v_{x}, v_{y}\right) & +\tilde{\psi}_{10}\left(v_{x}-v_{x c}, v_{y}-v_{y c}\right)+ \\
& +\overline{\tilde{\psi}}_{10}\left(v_{x}+v_{x c}, v_{y}+v_{y c}\right),
\end{aligned}
$$

whereas

$$
\tilde{I}_{10}\left(v_{x}, v_{y}\right)=\mathcal{F}\left\{I_{10}(x, y)\right\}=\iint I_{10}(x, y) \exp \left[2 \pi i\left(v_{x} x+v_{y} y\right)\right] \mathrm{d} x \mathrm{~d} y,
$$

In eq. (21) $\tilde{I}_{d c}\left(v_{x}, v_{y}\right)$ is the contribution to the spectrum given by the low-frequency background illumination (Cuche et al., 2000; Liu et al., 2002; Onural, 2000), corresponding to a peak at low spatial frequencies; $\tilde{\psi}_{10}\left(v_{x}-v_{x c}, v_{y}-v_{y c}\right)$ and $\overline{\tilde{\psi}}_{10}\left(v_{x}+v_{x c}, v_{y}+v_{y c}\right)$ represent the Fourier spectrum of the vortex field and its complex conjugate, respectively, shifted by the carrier spatial frequencies $v_{x c}$ and $v_{y c}$ along the $x$ - and $y$-direction, respectively. These two terms contribute to the two outer peaks of Fig. 10(c), and correspond to the positive and negative components of the vortex field spectrum. By taking the shifted inverse Fourier transform of $\tilde{\psi}_{10}\left(v_{x}-v_{x c}, v_{y}-v_{y c}\right)$, the amplitude of the vortex field can be determined, as shown in Fig 10(d), respectively.

The retrieved phase distribution in Fig. 11 shows a spiral profile with a discontinuity line typical of single topological charge vortex (White et al., 1991). We point out that the described method can be usefully employed for characterizing the vorticity of infrared beams of potential use in optical telecommunication applications, where the preservation of the purity of the mode along the propagation direction (Indebetouw, 1993) is a problem of crucial importance. 
(d)

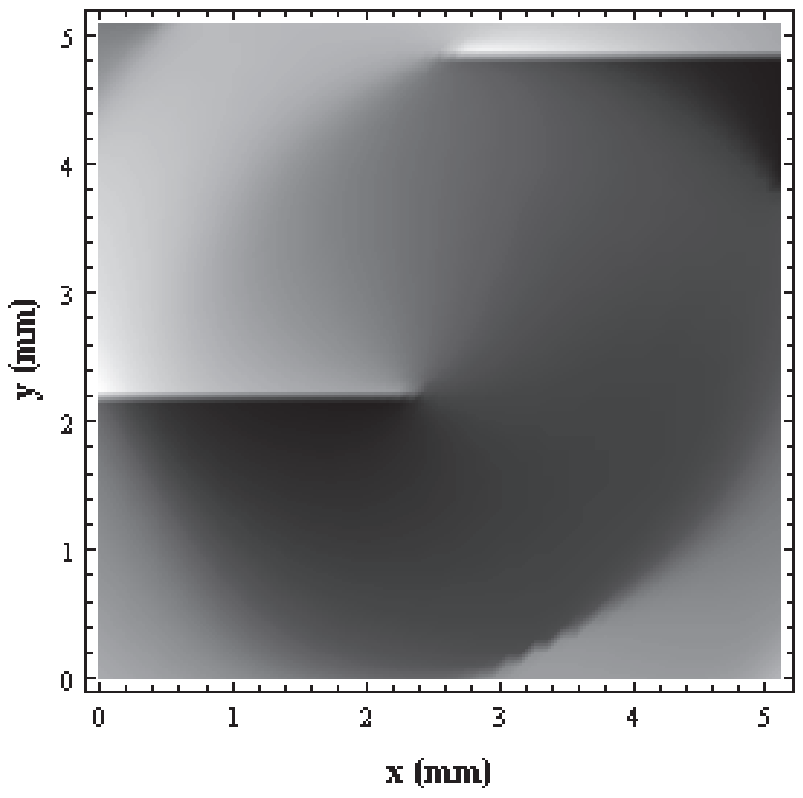

Fig. 11. Infrared Laguerre-Gaussian beam analysis based on Fresnel reconstruction of offaxis digital hologram: phase distribution of the lowest order vortex beam

\section{Conclusions}

In this chapter we have shown that the numerical reconstruction of a whole optical wavefield through digital holography can be successfully performed in the mid-infrared regime using pyroelectric and microbolometric sensors. Amplitude and phase reconstructions were obtained by back-Fresnel propagation from the hologram recording plane to the object plane. Digital holography is closely related to digital image processing and to the mathematical models of imaging. We have described methods for improving the accuracy of the reconstruction which allows us to compensate for the loss of resolution at longer wavelength and the low spatial resolution of the pyroelectric camera array. It is worth pointing out that the improved spatial resolution of digital holography in the mid infrared regime is a significant improvement in a number of biologically relevant measurements related to biological cell and tissue analysis, where electric potential or light induced phase changes are expected to play a significant role in the characterization of complex biological structures. Infrared digital holography has also been applied for large object investigation.

\section{References}

Allaria, E., Brugioni, S., De Nicola, S., Ferraro, P., Grilli, S. \& Meucci, R. (2003). Digital Holography at $10.6 \mu \mathrm{m}$. Optics Communications, Vol. 215, pp. 257-262. 
Allen, L., Beijersbergen, M.W., Spreeuw, R.J.C. and Woerdman, J.P. (1992). Orbital angular momentum of light and the transformation of Laguerre-Gaussian laser modes. Phys. Rev. A, Vol. 45, pp. 8185-8189.

Allen, M.L., Padgett, M.J. \& Babiker, M. (1999). The orbital angular momentum of light. Progress in Optics (Vol. 39), Emil Wolf (Elsevier), pp. 291-372, Amsterdam, Netherlands.

Arecchi, F.T., Giacomelli, G., Ramazza, P. L. \& Residori, S. (1991). Vortices and defects statistics in two dimensional optical chaos. Phys. Rev. Lett., Vol. 67, pp. 3749-3751.

Cuche, E., Marquet, P. \& Depeursinge, C. (1999a). Simultaneous amplitude-contrast and quantitative phase-contrast microscopy by numerical reconstruction of Fresnel offaxis holograms. Appl. Opt., Vol. 38, pp. 6994-7001.

Cuche, E., Bevilacqua, F. \& Depeursinge, C. (1999a). Digital Holography for quantitative phase-contrast imaging. Opt. Lett., Vol. 24, pp. 291-293 .

Cuche, E., Marquet. P. \& Depeursinge, C. (2000). Spatial filtering for zero-order and twinimage elimination in digital off-axis holography. Appl. Opt., Vol. 39, pp. 4070-4075.

Demoli, N., Vukicevic, D. \& Torzynski, M. (2003). Dynamic digital holographic interferometry with three wavelengths. Opt. Express, Vol. 11, pp. 767-774.

De Nicola, S., Ferraro, P., Grilli, S., Miccio, L., Meucci, R., Buah-Bassuah, P.K. \& Arecchi, F.T. (2008). Infrared digital refelective-holographic 3D shape measurements. Opt. Commun., Vol. 281, pp. 1445-1449.

De Nicola, S., Zito, G., Meucci, R., Buah-Bassuah, P.K. \& Arecchi, F.T. (2010). Fourier projection method for measuring the two-point correlation of Laguerre-Gaussian modes. J. of Optics, Vol. 12, pp. 035404-035412.

Dubois, F., Joannes, L., Dupont, O., Dewandel, J.L. \& Legros, J.C. (1999). An integrated optical set-up for fluid-physics experiments under microgravity conditions. Meas. Sci. Technol., Vol. 10, pp. 934-945.

Gabor, D. (1948). A new microscopic principle. Nature, Vol. 161, pp. 777-778.

Gass, J., Dakoff, A. \& Kim, M.K. (2003). Phase imaging without $2 \pi$ ambiguity by multiwavelength digital holography. Optics Letters, Vol. 28, pp. 1141-1143.

Goodman, J.W. \& Lawrence, R.W. (1967). Digital image formation from electronically detected holograms. Appl. Phy. Lett., Vol. 11, pp. 77-79.

Goodman, J.W. (1996). Introduction to Fourier Optics (Ed. 2). McGraw-Hill, New York, USA.

Grilli, S., Ferraro, P., De Nicola, S., Finizio, A., Pierattini, G. \& Meucci, R. (2001). Whole optical wavefields reconstruction by digital holography. Opt. Exp., Vol. 9, pp. 294-302.

Indebetouw, G. (1993). Optical vortices and their propagation. J. Mod. Opt., Vol. 40, pp. 73-87.

Jueptner, W.P., Werner, P., Kujawinska, M., Osten, W., Salbut, L.A. \& Seebacher, S. (1987). Combined measurement of silicon microbeams by grating interferometry and digital holography. Proceeding of SPIE International Conference on Applied Optical Metrology, P. K. Rastogi and F. Gyimesi Eds., Vol. 3407, pp. 348-357.

Kato, J., Yamaguchi, I. \& Matsumura, T. (2003). Multicolor digital holography with an achromatic phase shifter. Opt. Lett., Vol. 27, pp. 1403-1405.

Kim, M. (2000). Tomographic three-dimensional imaging of a biological specimen using wavelength-scanning digital interference holography. Opt. Express, Vol. 7, pp. 305310.

Kreis, T.M. \& Jüptner, W. (1997). Principles of Digital Holography. Jüptner and Osten Eds., Fringe 97, Academic Verlag, pp. 253-363. 
Kreis, T.M. (2002a). Frequency analysis of digital holography. Opt. Eng., Vol. 41, pp. 771-778.

Kreis, T.M. (2002b). Frequency analysis of digital holography with reconstruction by convolution. Opt. Eng., Vol. 41, pp. 1829-1839.

Kronrod, R.W., Merzlyakov, N.S. \& Yaroslavskii, L.P. (1972). Reconstruction of a hologram with a computer. Sov Phys Tech Phys., Vol. 17, pp. 333-334.

Lai, S., King, B. \& Neifeld, N.A. (2000). Wavefront reconstruction by means of phase-shifting digital in-line holography. Opt. Comm., Vol. 173, pp.155-160.

Leach, J., Padgett, M.J., Barnett, S.M., Franke-Arnold, S. \& Courtial, J. (2002). Measuring the orbital angular momentum of a single photon. Phys. Rev. Lett., Vol. 88, pp. 257901-04.

Lei, X., Xiaoyuan, P., Asundi, A.K. \& Jianmin, M. (2001a). Hybrid holographic microscope for interferometric measurement of microstructures. Opt. Eng., Vol. 40, pp. 2533-2539.

Lei, X., Xiaoyuan, P., Jianmin, M. \& Asundi, A.K. (2001b). Studies of Digital Microscopic Holography with Applications to Microstructure Testing. Appl. Opt., Vol. 40, pp. 5046-5052.

Leith, E. \& Upatnieks, J. (1965). Microscopy by wavefront reconstruction. J. Optical Society of America, Vol. 55, pp. 569-570.

Liu, C., Li, Y., Cheng, X., Liu, Z., Bo, F. \& Zhu, J. (2002). Elimination of zero-order diffraction in digital holography. Opt. Eng., Vol. 41, pp. 2434-2437.

Nilsson, B. \& Carlsson, T. (2000). Simultaneous measurement of shape and deformation using digital light-in-flight recording by holography. Opt. Eng., Vol. 39, pp. 244-253.

Onural, L. (2000). Sampling of the diffraction field. Appl. Opt., Vol. 39, pp. 5929-5935.

Paturzo, M., Pelagotti, A., Finizio, A., Miccio, L., Locatelli, M., Geltrude, A., Meucci, R. \& Ferraro, P. (2010). Optical reconstruction of digital holograms recorded at $10 \mu \mathrm{m}$ : a route for 3D imaging at long IR wavelengths. Optics Letters, Vol. 35, pp. 2112-2114.

Pedrini, G., Tiziani, H.J. \& Zoa, Y. (1996). Speckle size of digitally reconstructed wavefronts of diffusely scattering objects. J. Mod. Opt., Vol. 43, pp. 395-407.

Pedrini, G., Schedin, S. \& Tiziani, H.J. (2001). Aberration compensation in digital holographic reconstruction of microscopic objects. J. Mod. Opt., Vol. 48, pp. 1035-1041.

Pelagotti, A., Locatelli, M., Geltrude, A., Poggi, P., Meucci, R., Paturzo, M., Miccio, L. \& Ferraro, P. (2010a). Reliability of 3D imaging by digital holography at long IR wavelength. Journal of Display Techonogy, Vol. 6, pp. 465-471.

Rastogi, P.K. (1994). Holographic Interferometry. Springer Verlag, Berlin, Germany.

Schnars, U. (1994). Direct phase determination in hologram interferometry with use of digitally recorded holograms. J. Opt. Soc. Am. A, Vol. 11, pp. 2011-2015.

Schanrs, U. \& Juptner, W. (1994). Direct recording of holograms by a CCD target and numerical reconstruction. Appl. Opt., Vol. 33, pp. 179-181.

Schnars, U. \& Juptner, W. (2002). Digital recording and numerical reconstruction of holograms. Meas. Sci. Technol., Vol. 13, pp. R85-R101.

Seebacker, S., Osten, W., Baumbach, T. \& Juptner, W. (2001). The determination of materials parameters of micro-components using digital holography. Opt. Las. Eng., Vol. 36, pp. 103-126.

Soskin, M. S. \& Vasnetov, M. V. (2001). Singular Optics. Progress in Optics (Vol. 42), Emil Wolf (Elsevier), pp. 219-276, Amsterdam, Netherlands.

Stetson, K.A. \& Powell, R.L. (1966). Hologram Interferometry. J. Optical Society of America, Vol. 54, pp. 1161-1166. 
Stetson, K.A. \& Brohinsky, W.R. (1985). Electrooptic holography, its application to hologram interferometry. App. Opt., Vol. 24, pp. 3631.

Vest, C.M. (1979). Holographic Interferometry. John Wiley, New York, USA.

White, A.G., Smith, C. P., Heckenberg, N.R., Rubinsztein-Dunlop, H., Mcduff, R. \& Weiss, C. O. (1991). Interferometric measurements of phase singularities in the output of a visible laser. J. Mod. Opt., Vol. 12, pp. 2531-41.

Yamaguchi, I. \& Zhang, T. (1997). Phase-shifting digital holography. Opt. Lett., Vol. 23. Pp. 1268-1270.

Yamaguchi, I., Matsumura, T. \& Kato, J. (2002). Phase-shifting colour digital holography. Optics Letters, Vol. 27, pp. 1108-1110.

Yaroslavsky, L. \& Eden, M. (1996). Fundamentals of Digital Optics. BirkhŠuser, Boston, USA.

Zhang, F., Yamaguchi, I. \& Yaroslavsky, L.P. (2004). Algorithm for reconstruction of digital holograms with adjustable magnification. Opt. Lett., Vol. 29, pp. 1668-1670.

Zito, G., Finizio, A. \& De Nicola, S. (2009). Spatially resolved refractive index profiles of electrically switchable computer-generated holographic gratings. Opt. Express, Vol. 17, pp. 18843-18851. 
ADVANCED HOLOGRAPHY METROLOGY AND IMAGING

Edace by trabele Napdanova

\author{
Advanced Holography - Metrology and Imaging \\ Edited by Dr Izabela Naydenova
}

ISBN 978-953-307-729-1

Hard cover, 374 pages

Publisher InTech

Published online 09, November, 2011

Published in print edition November, 2011

Advanced Holography - Metrology and Imaging covers digital holographic microscopy and interferometry, including interferometry in the infra red. Other topics include synthetic imaging, the use of reflective spatial light modulators for writing dynamic holograms and image display using holographic screens. Holography is discussed as a vehicle for artistic expression and the use of software for the acquisition of skills in optics and holography is also presented. Each chapter provides a comprehensive introduction to a specific topic, with a survey of developments to date.

\section{How to reference}

In order to correctly reference this scholarly work, feel free to copy and paste the following:

Sergio De Nicola, Andrea Geltrude, Massimiliano Locatelli, Kais Al-Naimee, Riccardo Meucci and F.Tito Arecchi (2011). Infrared Holography for Wavefront Reconstruction and Interferometric Metrology, Advanced Holography - Metrology and Imaging, Dr Izabela Naydenova (Ed.), ISBN: 978-953-307-729-1, InTech, Available from: http://www.intechopen.com/books/advanced-holography-metrology-and-imaging/infraredholography-for-wavefront-reconstruction-and-interferometric-metrology

\section{INTECH}

open science | open minds

\section{InTech Europe}

University Campus STeP Ri

Slavka Krautzeka 83/A

51000 Rijeka, Croatia

Phone: +385 (51) 770447

Fax: +385 (51) 686166

www.intechopen.com

\section{InTech China}

Unit 405, Office Block, Hotel Equatorial Shanghai

No.65, Yan An Road (West), Shanghai, 200040, China

中国上海市延安西路65号上海国际贵都大饭店办公楼 405 单元

Phone: +86-21-62489820

Fax: +86-21-62489821 
(C) 2011 The Author(s). Licensee IntechOpen. This is an open access article distributed under the terms of the Creative Commons Attribution 3.0 License, which permits unrestricted use, distribution, and reproduction in any medium, provided the original work is properly cited. 